\title{
Letter to the Editor Regarding "Surgical Management of Recurrent and Persistent Macular Holes: A Practical Approach"
}

\author{
Thibaud Garcin (D)
}

Received: January 8, 2022 / Accepted: February 8, 2022 / Published online: February 25, 2022

(C) The Author(s) 2022

Keywords: Complex Macular Hole; Epiretinal; Lyophilized Human Amniotic Membrane; Overlay; Rhegmatogenous Retinal Detachment

\section{Key Summary Points}

\section{Why carry out this letter?}

The review presented by Cao et al. discussed current approaches to manage recurrent or persistent macular holes, without mentioning in detail all surgical technique variations of human amniotic membrane use.

\section{What was learned from the letter?}

Human amniotic membrane can be either cryopreserved or lyophilized according to availability in various countries or surgeon personal practices.

T. Garcin

Ophthalmology Department, University Hospital, Saint-Étienne, France

T. Garcin $(\bowtie)$

Corneal Graft Biology, Engineering and Imaging Laboratory, BiiGC, EA2521, Federative Institute of Research in Sciences and Health Engineering, Faculty of Medicine, Jean Monnet University, 10, Rue de la Marandière, 42055 Saint-Étienne Cedex 2, France

e-mail: t.garcin@univ-st-etienne.fr
Human amniotic membrane has been increasingly used and reported as inlay (i.e., subretinally positioned inside/lying on the macular hole with the "chorion down"). Nevertheless, overlay (i.e., epiretinal position with "chorion up") should be considered as a valuable option: it provides encouraging anatomical and functional midterm results, while offering numerous advantages (safer, no additional trauma of the foveal area, shorter surgery, retinal layer organization respected, potential reversibility) compared to inlay.

Cao et al. have published "Surgical Management of Recurrent and Persistent Macular Holes: A Practical Approach" [1]. This relatively exhaustive review presents current options to manage complex macular holes (MHs) after primary failure, especially one of the most recent developments, human amniotic membrane (AM) transplantation.

However, it would be interesting to discuss surgical techniques using this adjuvant to close complex MHs. Indeed, three key points must be developed: the nature of AM, the position of $\mathrm{AM}$, and the orientation of AM. Thus, we discuss additional references which could bring the readers a more precise overview of the issues when AM is used to promote $\mathrm{MH}$ closure. 
Different techniques using cryopreserved AM (cAM), a widely available tissue, have provided encouraging results either used first as a plug transplanted into the subretinal space [2-6] or placed secondly in an epiretinal position [7]. Rizzo et al. [2-4] used cAM from a local eye bank (Lucca, Italy) and positioned the plug with "chorion down", facing the retinal pigment epithelium (RPE) (i.e., as an inlay [8]). Abouhussein et al. [5] used homemade cAM and Huang et al. [6] used cAM from AmnioGraft (Bio-Tissue, Miami, FL, USA) transplanted as inlay. This chorion-down orientation of the plug of cAM subretinally transplanted may ensure proper adhesion on the RPE, preventing secondary displacement. Moharram et al. [7] did not specify the source of their cAM, and were the only team who reported epiretinal use of cAM to close $\mathrm{MH}$-associated rhegmatogenous retinal detachment (RRD) in highly myopic eyes: their rationale was to consider complex $\mathrm{MH}$ as a macular ulcer by analogy with persistent corneal ulcers. They positioned the cAM plug with the chorion down, facing the retina, therefore not as a "true" overlay as defined by Letko et al. [8] for corneal applications. Note that population samples in this series using cAM were small, and comparability was limited by lack of data regarding mean preoperative $\mathrm{MH}$ diameter or heterogeneous baseline characteristics and follow-up.

Lyophilized AM (lAM) was used for the first time in ophthalmology in 2004 [9], with similar physical, biological, and structural properties to cAM [10]. Compared to cAM, IAM presents several advantages: immediate availability in the operating room with simpler logistics [11]; long shelf life at room temperature; thinner and more transparent [6], which can help in integrating it when used as an inlay, or as a smart interface with less mask effect when used as an overlay; and easy to trephine before rehydration, with roll-up allowing a "no touch" technique [12] for lAM insertion thanks to a dedicated catheter.

We recently published [12] an interesting standardized surgical technique which combines the advantages of IAM and the epiretinal position with "chorion up" (i.e., lAM used as a "true" overlay [8]). We used sterile devitalized trephined discs of IAM (Visio Amtrix, TBF, Mions, France) with "chorion up" to cover the MH with ample overlap for easier handling and positioning. The rationale combines mutually nonexclusive hypotheses: (1) The overlay can play the same role as an inverted internal limiting membrane (ILM) flap [13], but will be larger, easier to position, and more stable. Like a biological bandage, it can act as a scaffold to promote healing, with centripetal migration of cells, stimulation of macrophage-like cells facilitating $\mathrm{MH}$ closure, and a more physiologic closure mechanism versus subretinal position [14]; besides, if complete closure is impossible, it acts as a patch and prevents $\mathrm{MH}$-induced RRD. We hoped to obtain excellent functional results by analogy with those already obtained for ILM used as an epiretinal inverted flap versus insertion into the MH [15]: the epiretinal position resulted in significantly better recovery of photoreceptor layers, and therefore better visual recovery. (2) The overlay better respects the organization of all retinal layers, preventing induction of foveal gliosis by interposition of exogenous tissue (cAM or lAM) transplanted into the subretinal space, which must be integrated between the $\mathrm{MH}$ edges. (3) It seemed safer not to manipulate the MH edges, so as not to worsen the RPE and neuroretinal injuries, particularly during graft insertion [16]. (4) The overlay could prevent the parafoveal atrophy described after retraction of CAM or lAM used as inlays [17]. (5) Even considering the time taken to fully unfold the IAM for overlay, operating time can be shortened versus inlay, thus reducing light toxicity [18]. (6) If an adverse event occurs, the lAM can be removed, which is a key point for a new technique.

In our series of complex $\mathrm{MH}$ cases with no alternative [12] (minimum and maximum diameters, respectively $945 \pm 330$ and $1507 \pm 717 \mu \mathrm{m}$; axial length $26.58 \pm 3.38 \mathrm{~mm}$; number of prior surgeries $1.4 \pm 0.96$ ), the overlaid epiretinal large disc of ILM blue-stained lAM with the chorion up seemed to promote anatomic success $(80 \%$ of $\mathrm{MH}$ closed, $20 \%$ had reduced diameter, all $\mathrm{MH}$-associated RRD reattached without recurrence) and functional recovery (mean logMAR BCVA improved from $1.92 \pm 0.58$ to $1.17 \pm 0.57 ; P<0.001$ ), with 
$90 \%$ of eyes achieving $\geq 0.3 \log$ MAR improvement) with 1-year follow-up. Thus, lAM used as overlay should be considered as a valuable, promising minimally invasive technique among the options to close recurrent or persistent MHs.

Indeed, large samples with homogeneous baseline characteristics and long follow-up must now be investigated, ideally in randomized multicentric studies that should compare these techniques to one another, for example lAM inlays with the chorion down and overlays with the chorion up, using our protocol.

\section{ACKNOWLEDGEMENTS}

Funding. No funding or sponsorship was received for this study or publication of this article.

Authorship. All named authors meet the International Committee of Medical Journal Editors (ICMJE) criteria for authorship for this article, take responsibility for the integrity of the work as a whole, and have given their approval for this version to be published.

Author Contributions. Conceptualization, methodology, formal analysis and investigation, writing-original draft preparation, writing-review and editing, resources, supervision: Thibaud Garcin.

Compliance with Ethics Guidelines. This article is based on previously conducted studies and does not contain any new studies with human participants or animals performed by any of the authors.

Disclosures. Thibaud Garcin has no conflicts of interest to declare.

Data availability. Data sharing is not applicable to this article as no datasets were generated or analyzed during the current study.

Open Access. This article is licensed under a Creative Commons Attribution-
NonCommercial 4.0 International License, which permits any non-commercial use, sharing, adaptation, distribution and reproduction in any medium or format, as long as you give appropriate credit to the original author(s) and the source, provide a link to the Creative Commons licence, and indicate if changes were made. The images or other third party material in this article are included in the article's Creative Commons licence, unless indicated otherwise in a credit line to the material. If material is not included in the article's Creative Commons licence and your intended use is not permitted by statutory regulation or exceeds the permitted use, you will need to obtain permission directly from the copyright holder. To view a copy of this licence, visit http:// creativecommons.org/licenses/by-nc/4.0/.

\section{REFERENCES}

1. Cao JL, Kaiser PK. Surgical management of recurrent and persistent macular holes: a practical approach. Ophthalmol Ther. 2021;10(4):1137-53.

2. Rizzo S, Caporossi T, Tartaro R, Finocchio L, Franco F, Barca F, et al. A human amniotic membrane plug to promote retinal breaks repair and recurrent macular hole closure. Retina. 2019;39(Suppl 1): S95-103.

3. Caporossi T, De Angelis L, Pacini B, Tartaro R, Finocchio L, Barca F, et al. A human Amniotic Membrane plug to manage high myopic macular hole associated with retinal detachment. Acta Ophthalmol. 2020;98(2):e252-6.

4. Caporossi T, Pacini B, De Angelis L, Barca F, Peiretti E, Rizzo S. Human amniotic membrane to close recurrent, high myopic macular holes in pathologic myopia with axial length of $>/=30 \mathrm{~mm}$. Retina. 2020;40(10):1946-54.

5. Abouhussein MA, Elbaha SM, Aboushousha M. Human amniotic membrane plug for macular holes coexisting with rhegmatogenous retinal detachment. Clin Ophthalmol. 2020;14:2411-6.

6. Huang YH, Tsai DC, Wang LC, Chen SJ. Comparison between cryopreserved and dehydrated human amniotic membrane graft in treating challenging cases with macular hole and macular hole retinal detachment. J Ophthalmol. 2020;2020:9157518. 
7. Moharram HM, Moustafa MT, Mortada HA, Abdelkader MF. Use of epimacular amniotic membrane graft in cases of recurrent retinal detachment due to failure of myopic macular hole closure. Ophthalmic Surg Lasers Imaging Retina. 2020;51(2):101-8.

8. Letko E, Stechschulte SU, Kenyon KR, Sadeq N, Romero TR, Samson CM, et al. Amniotic membrane inlay and overlay grafting for corneal epithelial defects and stromal ulcers. Arch Ophthalmol. 2001;119(5):659-63.

9. Nakamura T, Yoshitani M, Rigby H, Fullwood NJ, Ito $\mathrm{W}$, Inatomi $\mathrm{T}$, et al. Sterilized, freeze-dried amniotic membrane: a useful substrate for ocular surface reconstruction. Invest Ophthalmol Vis Sci. 2004;45(1):93-9.

10. Allen CL, Clare G, Stewart EA, Branch MJ, McIntosh OD, Dadhwal M, et al. Augmented dried versus cryopreserved amniotic membrane as an ocular surface dressing. PLoS ONE. 2013;8(10):e78441.

11. Libera RD, Melo GB, Lima Ade S, Haapalainen EF, Cristovam P, Gomes JA. Assessment of the use of cryopreserved $\mathrm{x}$ freeze-dried amniotic membrane (AM) for reconstruction of ocular surface in rabbit model. Arq Bras Oftalmol. 2008;71(5):669-73.

12. Garcin T, Gain P, Thuret G. Epiretinal large disc of blue-stained lyophilized amniotic membrane to treat complex macular holes: a 1-year follow-up. Acta ophthalmologica. 2021.
13. Boninska K, Nawrocki J, Michalewska Z. Mechanism of "Flap Closure" after the Inverted Internal Limiting Membrane Flap Technique. Retina. 2018;38(11):2184-9.

14. Tornambe PE. Macular hole genesis: the hydration theory. Retina. 2003;23(3):421-4.

15. Park JH, Lee SM, Park SW, Lee JE, Byon IS. Comparative analysis of large macular hole surgery using an internal limiting membrane insertion versus inverted flap technique. $\mathrm{Br} \mathrm{J}$ Ophthalmol. 2019;103(2):245-50.

16. Caporossi T, Tartaro R, Giansanti F, Rizzo S. The amniotic membrane for retinal pathologies. Insights on the surgical techniques. Graefe's archive for clinical and experimental ophthalmology = Albrecht von Graefes Archiv fur klinische und experimentelle Ophthalmologie. 2020;258(6): 1347-9.

17. Tsai DC, Huang YH, Chen SJ. Parafoveal atrophy after human amniotic membrane graft for macular hole in patients with high myopia. $\mathrm{Br} \mathrm{J}$ Ophthalmol. 2020;105:1002-10.

18. van den Biesen PR, Berenschot T, Verdaasdonk RM, van Weelden $H$, van Norren D. Endoillumination during vitrectomy and phototoxicity thresholds. $\mathrm{Br}$ J Ophthalmol. 2000;84(12):1372-5. 